\title{
Comparison Between Adenoviral and Retroviral Vectors for the Transduction of the Thymidine Kinase PET Reporter Gene in Rat Mesenchymal Stem Cells
}

\author{
Véronique Roelants ${ }^{1,2}$, Daniel Labar ${ }^{2}$, Carole de Meester ${ }^{1}$, Xavier Havaux ${ }^{1}$, Antonio Tabilio ${ }^{3}$, Sanjiv S. Gambhir ${ }^{4}$, \\ Mauro Di Ianni ${ }^{3}$, Anne Bol $^{2}$, Luc Bertrand ${ }^{1}$, and Jean-Louis Vanoverschelde ${ }^{1}$ \\ ${ }^{1}$ Université catholique de Louvain, Division of Cardiology, Brussels, Belgium; ${ }^{2}$ Université catholique de Louvain, IMRE Unit, \\ Louvain-la-Neuve, Belgium; ${ }^{3}$ Department of Clinical and Experimental Medicine, Perugia University, Perugia, Italy; and \\ ${ }^{4}$ Bio-X Program, Department of Radiology and Bioengineering, Stanford University School of Medicine, Stanford, California
}

\begin{abstract}
Mesenchymal stem cells (MSCs) are a promising cell line for the treatment of ischemic heart disease. To evaluate the success of their transplantation into living animals, noninvasive imaging techniques that are able to track the distribution and fate of those cells would be useful. The aim of this study was to investigate the feasibility of infecting rat MSCs with adenoviruses and retroviruses carrying the herpes simplex virus type 1 thymidine kinase (HSV1-tk) gene; to compare the level of transgene expression induced by the 2 viral vectors; to evaluate the effects of viral transduction on cell phenotype, viability, proliferation rates, and differentiation capabilities; and to test the possibility of noninvasively imaging transduced MSCs using 9-(4-18F-fluoro-3[hydroxymethyl]butyl)guanine $\left({ }^{18} \mathrm{~F}-\mathrm{FHBG}\right)$ and small-animal PET after their transplantation into living rats. Methods: We infected rat bone marrow MSCs with adenoviruses carrying the HSV1 mutant tk (Ad-HSV1-sr39tk) PET reporter gene (PRG) or with a retroviral construct expressing the wild-type HSV1-tk PRG. The efficacy and intensity of HSV1-sr39tk and HSV1-tk gene expression were determined by a direct comparison of $\left[8-{ }^{3} \mathrm{H}\right]$-penciclovir $\left(\left[8-{ }^{3} \mathrm{H}\right]-\mathrm{PCV}\right)$ cell uptake in both infected MSC populations and noninfected control MSCs. Small-animal PET studies were performed on living rats after an intramuscular injection of infected MSCs. The MSCs either have been incubated in advance with ${ }^{18} \mathrm{~F}-\mathrm{FHBG}$ or were administered after an intravenous injection of ${ }^{18}$ F-FHBG. Results: Both adenoviral and retroviral vectors can be used to introduce the tk PRG in MSCs. Neither adenovirus nor retrovirus infections significantly modify MSC phenotype, viability, proliferation, and differentiation capabilities. No significant ${ }^{3} \mathrm{H}-\mathrm{PCV}$ uptake was observed in noninfected MSCs. By contrast, after both adenoviral and retroviral infections, the infected MSC populations exhibited a similar, significantly higher, ${ }^{3} \mathrm{H}-\mathrm{PCV}$ accumulation. Small-animal PET images showed intense activity within the transplanted regions irrespective of the infected MSC population used. Conclusion: Our results demonstrate the feasibility of
\end{abstract}

Received Feb. 28, 2008; revision accepted Aug. 7, 2008.

For correspondence or reprints contact: Jean-Louis Vanoverschelde, Division of Cardiology, Cliniques Universitaires Saint-Luc, Université Catholique de Louvain, Ave. Hippocrate 10, 1200 Brussels, Belgium.

E-mail: vanoverschelde@card.ucl.ac.be

COPYRIGHT $\odot 2008$ by the Society of Nuclear Medicine, Inc. infecting MSCs with adenoviruses and retroviruses expressing the HSV1-tk PRG and suggest that infected MSCs can be noninvasively imaged with ${ }^{18} \mathrm{~F}-\mathrm{FHBG}$ and small-animal PET after their transplantation into living animals.

Key Words: mesenchymal stem cells; reporter gene imaging; small-animal PET

J Nucl Med 2008; 49:1836-1844

DOI: 10.2967/jnumed.108.052175

T nterest in mesenchymal stem cells (MSCs) for cardiac repair has increased exponentially during the past $5 \mathrm{y}$. Compared with other cell types considered for cardiomyoplasty, MSCs appear to possess unique properties that may allow for convenient and highly effective cell therapy. MSCs can be used allogenetically and delivered systemically and can differentiate into a cardiomyocytelike phenotype when implanted in healthy myocardium. Furthermore, MSCs can be readily transduced by a variety of vectors and maintain transgene expression after in vivo differentiation (1-5).

To evaluate the success of stem cell transplantation and to monitor its outcome longitudinally in living individuals require the development of noninvasive imaging modalities capable of identifying the location, magnitude, and duration of cellular survival and fate. To be applicable in humans, these methods should be biocompatible, safe, and nontoxic; should provide quantitative information on the number of cells present in the region of interest; and should not be influenced by cell proliferation. At present, no single imaging modality possesses all of these characteristics.

Wu et al. (6) have validated a reporter gene imaging method that allows transplanted rat embryonic cardiomyoblasts to be imaged noninvasively using $9-\left(4-{ }^{18}\right.$ F-fluoro-3[hydroxymethyl] butyl)guanine $\left({ }^{18} \mathrm{~F}-\mathrm{FHBG}\right)$ and PET. The method uses the mutant herpes simplex virus type 1 thymidine kinase gene (HSV1-sr39tk) as a reporter gene. The 
product of this gene, the enzyme thymidine kinase (tk), can phosphorylate labeled thymidine or guanosine analogs, which can serve as reporter probes. Once phosphorylated, these labeled probes are trapped intracellularly and can be detected with a $\beta$-counter or a PET camera.

Transduction of PET reporter genes into the cell line of interest is usually achieved using adenoviral vectors (7-9). Unfortunately, adenoviral transduction only leads to episomal gene expression, whereby the reporter gene is not integrated into the infected cells. Expression of the transgene is thus transient, which is a severe limitation for tracking stem cell survival and proliferation longitudinally. It was recently suggested that the use of retroviruses instead of adenoviruses could induce more stable transgene expression (10). Retroviral and lentiviral vectors indeed offer the opportunity to stably integrate their genetic material into the cell chromatin. Thus, in principle, they should allow for a more prolonged and more stable transgene expression and for the longitudinal tracking of the transduced cells. Accordingly, the aim of the present study was to investigate the feasibility of infecting rat MSCs with adenoviruses and retroviruses carrying the HSV1-tk gene; to test the effects of viral transduction on cell phenotype, viability, proliferation rates, and differentiation capabilities; to test the effect of the reporter probe ${ }^{18} \mathrm{~F}-\mathrm{FHBG}$ on cell viability and proliferation rates; to compare the level of transgene expression induced by the 2 viral vectors; and to test the possibility of noninvasively imaging transduced MSCs using the reporter probe ${ }^{18} \mathrm{~F}-\mathrm{FHBG}$ and small-animal PET after their transplantation into muscle of living rats.

\section{MATERIALS AND METHODS}

All animal procedures were conducted in strict compliance with the European Community Council Directive of November 24, 1986 (86-609/EEC), and Decree of October 20, 1987 (87-848/EEC).

\section{Isolation and Culture of MSCs}

Eight-week-old male Wistar rats (weight, 345-355 g) were obtained from the central animal facility of the Université catholique de Louvain and were euthanized with an overdose of pentobarbital solution. Both tibias and femurs were dissected free, the ends of the bones were cut, and the bone marrow was extruded in 5 $\mathrm{mL}$ of Dulbecco's modified Eagle's medium with a syringe. The cells were then plated in culture medium (Dulbecco's modified Eagle's medium supplemented with $10 \%$ fetal bovine serum, $85 \mu \mathrm{g}$ of streptomycin per milliter, and 85 units of penicillin per milliliter) and incubated at $37^{\circ} \mathrm{C}$ in a humidified atmosphere containing $5 \%$ carbon dioxide. Nonadherent cells were removed on a daily basis by replacing the medium. After $10-15 \mathrm{~d}$, a confluent spindleshaped, fibroblastlike population of MSCs was obtained. The cells were maintained in culture, trypsinized when $80 \%$ confluence was reached, and replated at a density of $5 \times 10^{3}$ cells $/ \mathrm{cm}^{2}$. By repeating this protocol, cultures could be maintained beyond passage 20 .

\section{Viral Infections}

Replication-defective recombinant adenoviruses carrying a cytomegalovirus promoter driving the mutant herpes simplex type 1 virus tk reporter gene (Ad-CMV-HSV1-sr39tk) were kindly pro- vided by Sanjiv S. Gambhir (11). Cells producing an LSN-tk retroviral vector derived from the Moloney murine leukemia virus were kindly provided by Antonio Tabilio. This vector contains the HSV1-tk gene controlled by the long terminal repeat promoter and the neomycin resistance (neoR) gene transcribed from the early promoter of an internal simian virus 40 (12).

Adenoviral infection with Ad-CMV-HSV1-sr39tk was performed as previously described (13). Briefly, MSCs between passages 3 and 8 were incubated with various Ad-CMV-HSV1sr39tk viral titers (multiplicity of infection [MOI], 0, 5, 10, 20, 40, and 80). The adenovirus-infected cells were called Ad-sr39tkMSCs and were always used $24 \mathrm{~h}$ after infection.

Retroviral infection was induced using cells producing the retroviral vector as previously described (12). Briefly, the viral supernatant of the producing cells was added to the MSC (between passages 3 and 8) culture medium, supplemented with polybrene $(8 \mu \mathrm{g} / \mathrm{mL})$ in a $1: 1$ ratio after filtration through a $0.45-\mu \mathrm{m}$-pore filter. The cells were left for $24 \mathrm{~h}$ in the incubator. A second infection was then induced, and cells were cultured for $10 \mathrm{~d}$ in a medium supplemented with G-418 sulfate $(0.6 \mathrm{mg} / \mathrm{mL}$; Invitrogen Corp.) to finally obtain an MSC population expressing the neoR and tk genes. This population was called Ret-tk-MSCs. Control experiments in which noninfected MSCs underwent the same protocol were always conducted in parallel; all noninfected MSCs died within 7-8 d. To ensure that no cell lacking the neoR gene would survive, exposure to G-418 sulfate was always continued for 2 more days (up to $10 \mathrm{~d}$ ).

\section{Effect of Reporter Genes on MSC Phenotype, Viability, Proliferation, and Differentiation Potential}

To characterize their phenotype, MSCs, Ad-sr39tk-MSCs, and Ret-tk-MSCs at passages $1,5,10$, and 20 were fixed in $4 \%$ paraformaldehyde and $0.2 \%$ Triton X-100 (Sigma-Aldrich). After the cells were washed, they were incubated with primary mouse antibodies directed against either the rat CD45 (BD Pharmingen) or the CD90 (Cymbus Biotechnology) surface antigens. A secondary antibody was then introduced (donkey antimouse [Alexa Fluor 594; Molecular Probes]), and the cells were washed and examined under a fluorescence microscope (BX60; Olympus). In addition to this qualitative characterization, the proportion of CD45- and CD90positive cells was also analyzed using flow cytometry (FACScan; Becton Dickinson). For this purpose, cells at passage 3 were used. After trypsinization, $2 \times 10^{5}$ cells were resuspended in $100 \mu \mathrm{L}$ of phosphate-buffered saline (PBS) and incubated with antibodies directed against either the CD45 (fluorescein isothiocyanate rat antimouse CD45; BD Biosciences) or the CD90 (phycoerythrin mouse antirat CD90; BD Biosciences) antigens for $30 \mathrm{~min}$. The cells were then washed with PBS, fixed in CellFix (BD Biosciences), and analyzed on the flow cytometer. Data were generated using the CELLQUEST software (Becton Dickinson).

The effect of introducing the reporter gene on MSC viability and proliferation capabilities was tested using the trypan blue exclusion assay and the DHL cell proliferation assay kit (Anaspec), respectively.

To test the differentiation potential of our cell lines, MSCs, Adsr39tk-MSCs, and Ret-tk-MSCs were cultured in the presence of specific differentiation medium. To induce adipogenic differentiation, the cells were first plated at a density of $2 \times 10^{4}$ cells $/ \mathrm{cm}^{2}$ and incubated in our basic culture medium until reaching $100 \%$ confluence. At this point, the culture medium was supplemented with $1 \mu \mathrm{M}$ dexamethasone, $0.2 \mathrm{mM}$ indomethacine, $10 \mu \mathrm{g}$ of insulin per 
milliliter, and $0.5 \mathrm{mM}$ isobutylmethylxanthine (induction medium), and incubation was continued for an additional $3 \mathrm{~d}$. After this period, the induction medium was replaced by a maintenance medium (culture medium supplemented with a $10 \mu \mathrm{g} / \mathrm{mL}$ concentration of insulin) in which the cells were incubated for another $3 \mathrm{~d}$. These last 2 steps were repeated 5 times over a 30-d period. After completion of the adipogenic differentiation protocol, the cells were stained with Oil Red O (MP Biochemicals) to demonstrate the accumulation of lipid droplets in differentiated cells.

Osteogenic differentiation was induced by maintaining cells plated at a density of $3 \times 10^{3}$ cells $/ \mathrm{cm}^{2}$ in a basic culture medium supplemented with $0.1 \mu \mathrm{M}$ dexamethasone, $50 \mu \mathrm{M}$ ascorbic acid, and $10 \mathrm{mM} \beta$-glycerophosphate. The cells were incubated for a total of $3 \mathrm{wk}$, during which time the differentiation medium was replaced every $3 \mathrm{~d}$. After completion of the osteogenic differentiation protocol, von Kossa staining (UCB S.A.) was used to demonstrate the presence of calcium deposits in between differentiated cells.

\section{Effect of Reporter Probe on MSC Viability and Proliferation}

Ad-sr39tk-MSCs and Ret-tk-MSCs were incubated for $3 \mathrm{~h}$ and $30 \mathrm{~min}$ with ${ }^{18} \mathrm{~F}$-FHBG $(185 \mathrm{MBq}$ in $5 \mathrm{~mL})$. The effect of the reporter probe on the viability and proliferation capabilities of MSCs was tested using the trypan blue exclusion assay and the DHL cell proliferation assay kit (Anaspec), $3 \mathrm{~d}$ after incubation with the reporter probe.

\section{In Vitro Assay for HSV1-tk and HSV1-sr39tk Enzyme Activity}

HSV1-tk and HSV1-sr39tk enzyme activity was evaluated by measuring the rate of ${ }^{3} \mathrm{H}$-penciclovir $\left({ }^{3} \mathrm{H}-\mathrm{PCV}\right.$ ) (Moravek Biochemicals) incorporation into cells. For this purpose, Ad-sr39tkMSCs, Ret-tk-MSCs, and control nontransfected MSCs were incubated in the presence of a fixed amount $(37 \mathrm{kBq} / \mathrm{mL}[21.1$ $\mathrm{Ci} / \mathrm{mmol}])$ of ${ }^{3} \mathrm{H}-\mathrm{PCV}$ for $30,60,90,120$, and $180 \mathrm{~min}$ or in the presence of increasing doses (of 12, 37, 111, and $370 \mathrm{kBq} / \mathrm{mL}$ ) of ${ }^{3} \mathrm{H}-\mathrm{PCV}$ for $60 \mathrm{~min}$. At the end of the incubation period, the medium was removed to measure total radioactivity using a scintillation counter (Tri-Carb 2900TR; Perkin Elmer). The cells were then washed 3 times with PBS and harvested to determine the cell-associated radioactivity. Results were expressed as the percentage conversion of ${ }^{3} \mathrm{H}-\mathrm{PCV}$ per milligram of protein or as the conversion of ${ }^{3} \mathrm{H}-\mathrm{PCV}$ in moles per milligram of protein.

\section{Time Course of tk Activity After Adenoviral or Retroviral Infection}

The cellular uptake of ${ }^{3} \mathrm{H}-\mathrm{PCV}$ was evaluated at $8,24,48$, and 72 $\mathrm{h}$ and $6 \mathrm{~d}$ after infection in Ad-sr39tk-MSCs and every month after infection, over a 3-mo period, in Ret-tk-MSCs, as described above.

\section{In Vitro and In Vivo PET of MSCs}

To determine the detection limits of our small-animal PET camera (Mosaic; Philips) (Supplemental Appendix A; supplemental materials are available online only at http://jnm.snmjournals. org), a first set of in vitro experiments was conducted in which decreasing amounts (from $5 \times 10^{5}$ to $1 \times 10^{3}$ ) of Ad-sr39tk-MSCs, infected for $24 \mathrm{~h}$, were plated in a 12-well dish. The day after plating, the cells were incubated for $1 \mathrm{~h}$ with ${ }^{18} \mathrm{~F}-\mathrm{FHBG}(37 \mathrm{MBq} / \mathrm{mL})$ (Supplemental Appendix B), washed with PBS, and imaged with small-animal PET for $10 \mathrm{~min}$. Before reconstruction, raw data were corrected for radioactive decay, random (delayed coincidence window technique) and scattered (tail-fitting algorithm) coincidences, and deadtime losses. All images were reconstructed with a fully 3-dimensional iterative algorithm.

To evaluate the ability of our PET system to detect infected cells in vivo, ${ }^{18} \mathrm{~F}$-FHBG-preincubated MSCs were injected into the anterior tibial muscle of 12 male Wistar rats; a muscular lesion was created by direct intramuscular injection of the Naja Mossambica cardiotoxin (Sigma) $(20 \mu \mathrm{g} / 100 \mathrm{~g}$ diluted in $20 \mu \mathrm{L})$. Twenty-four hours later, 3 to $6 \times 10^{6} \mathrm{Ad}$-sr39tk-MSCs $(n=8)$ or Ret-tk-MSCs $(n=4)$ were incubated during $1 \mathrm{~h}$ with ${ }^{18} \mathrm{~F}-\mathrm{FHBG}(199 \pm 16 \mathrm{MBq}$ in $5 \mathrm{~mL}$ ). The preincubated cells were then washed, diluted in 50 $\mu \mathrm{L}$ of PBS, and injected around the muscular lesion. Two emission images of $10 \mathrm{~min}$ each were acquired at $30 \mathrm{~min}$ and $4 \mathrm{~h}$ later as described above. After acquisition of the emission data, a transmission scan was performed in a single mode using a rotating 370$\mathrm{MBq}{ }^{137} \mathrm{Cs}$ source placed outside the field of view. To determine the site of the ${ }^{18} \mathrm{~F}-\mathrm{FHBG}$ activity, color-coded coronal emission images were superimposed on inverted gray-scaled transmission images. After the imaging session, the rats were euthanized and their anterior tibial muscles were harvested and placed into a well automatic $\gamma$-counter (1480 Wizard 3"; Wallac/PerkinElmer). To determine the minimal number of ${ }^{18} \mathrm{~F}-\mathrm{FHBG}$ prelabeled Adsr39tk-MSCs that can be detected by the small-animal PET camera, additional experiments were conducted in which an increasing number of labeled Ad-sr39tk-MSCs (from $1 \times 10^{4}$ to $4 \times 10^{6}$ cells) was injected into the tibial anterior muscle and then imaged, as described above.

Finally, we evaluated the possibility of imaging non-prelabeled MSCs after the intravenous administration of ${ }^{18} \mathrm{~F}-\mathrm{FHBG}$. For this purpose, rats that had received an intramuscular injection of $1 \times 10^{6}$ Ad-sr39tk-MSCs $(n=6)$ or Ret-tk MSCs $(n=3)$ in the triceps brachialis muscle were given an intravenous injection of ${ }^{18} \mathrm{~F}-\mathrm{FHBG}$ $(37 \pm 4 \mathrm{MBq}$ ) the day after MSC injection. PET was performed at 3 $\mathrm{h}$ after the intravenous administration of the label.

\section{Statistical Analysis}

Data are reported as mean $\pm 1 \mathrm{SD}$. Group comparisons were performed with a Student $t$ test or 1-way ANOVA where appropriate. The relationship between the muscular activity calculated with the small-animal PET and either the muscular activity measured in the well $\gamma$-counter or the number of injected cells was compared with linear regression. $P$ values of less than 0.05 were considered statistically significant.

\section{RESULTS}

\section{Assessment of Optimal Multiplicity of Adenoviral Infection}

MSCs were incubated with various titers of Ad-CMVHSV1-sr39tk. A progressive increase in the cellular uptake of ${ }^{3} \mathrm{H}-\mathrm{PCV}$ by the transfected MSCs was seen when the MOI was increased. This increase reached a plateau at a MOI of 40, thus suggesting that 40 was the optimal MOI to be used for the adenoviral infection (data not shown).

\section{Effect of Viral Infections on MSC Phenotype, Viability, Proliferation, and Differentiation Potential}

In medium containing fetal bovine serum, native, noninfected MSCs typically present as adherent fibroblastlike cells. These cells express the CD90 surface antigen and lack the CD45 surface antigen. To evaluate whether viral transduction 
modifies cell phenotype, we evaluated the morphology and the pattern of expression of the CD45 and CD90 surface antigens in Ad-sr39tk-MSCs and Ret-tk-MSCs at several passages. As shown in Figure 1A, the typical fibroblastlike morphology of MSCs and the expression of the CD90 surface antigen were maintained after transduction with both vectors. The presence of CD90 and the absence of CD45 were confirmed by fluorescent-activated cell sorting analysis (Fig. 1B).

Similarly, the trypan blue cell viability and the cell proliferation assays did not show any significant differences among noninfected MSCs, Ad-sr39tk-MSCs, or Ret-tkMSCs at 24 and $48 \mathrm{~h}$ after plating (Figs. 2A and 2B; $P=$ not significant for all comparisons).

Finally, as shown in Figure 2C, the ability of MSCs to differentiate into adipogenic and osteogenic lineages when exposed to appropriate medium was maintained after viral transduction. When inducing the adipogenic differentiation, both transduced and nontransduced MSCs accumulated lipid droplets as detected by Oil Red O staining. Similarly, after osteogenic differentiation, the presence of calcium deposits could be evidenced by von Kossa staining in both transduced and nontransduced MSC cultures.

\section{Effect of Reporter Probe on MSC Viability and Proliferation}

To evaluate whether exposure of the transduced cells to the reporter probe modifies their viability and proliferation capabilities, transduced MSCs were incubated for $3 \mathrm{~h}$ and $30 \mathrm{~min}$ with ${ }^{18} \mathrm{~F}-\mathrm{FHBG}(37 \mathrm{MBq} / \mathrm{mL})$. Cell viability and proliferation capabilities were studied $3 \mathrm{~d}$ after irradiation. As shown in Figures 3A and 3B, no significant differences in cell viability and proliferation rates were observed between nonexposed MSCs and either Ad-sr39tkMSCs or Ret-tk-MSCs $(P=$ not significant for all comparisons).

\section{tk Activity in Transduced MSCs}

As shown in Figure 4A, the percentage conversion of ${ }^{3} \mathrm{H}$ PCV increased linearly over time in cell lines overexpress-
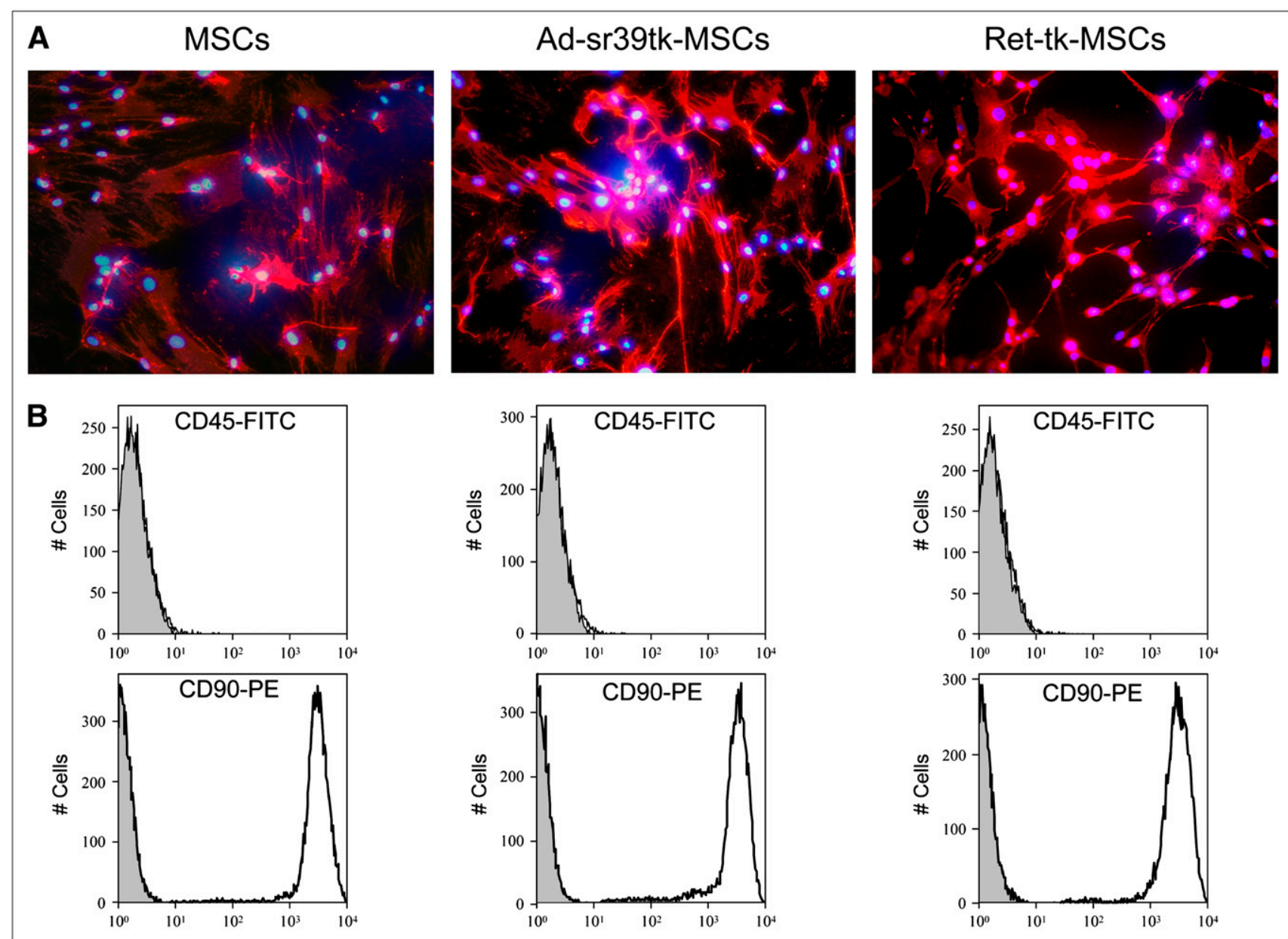

FIGURE 1. Phenotypic characterization of noninfected and infected MSCs. (A) Fluorescent micrographs show expression of CD90 surface antigen (red) by MSCs, Ad-sr39tk-MSCs, and Ret-tk-MSCs. Cell nuclei are stained in blue using 4',6'-diamidino-2phenylindole dihydrochloride. (B) Fluorescence-activated cell-sorting analysis of MSCs, Ad-sr39tk-MSCs, and Ret-tk-MSCs for CD45 and CD90 surface antigens. For CD45 antigen, fluorescent levels remained negligible in all $3 \mathrm{MSC}$ cell lines and in control cell population known to lack this antigen (gray). By contrast, for CD90 antigen all 3 MSC cell lines displayed high level of fluorescence when tested. FITC = phycoerythrin; pe = phycoerythrin. 


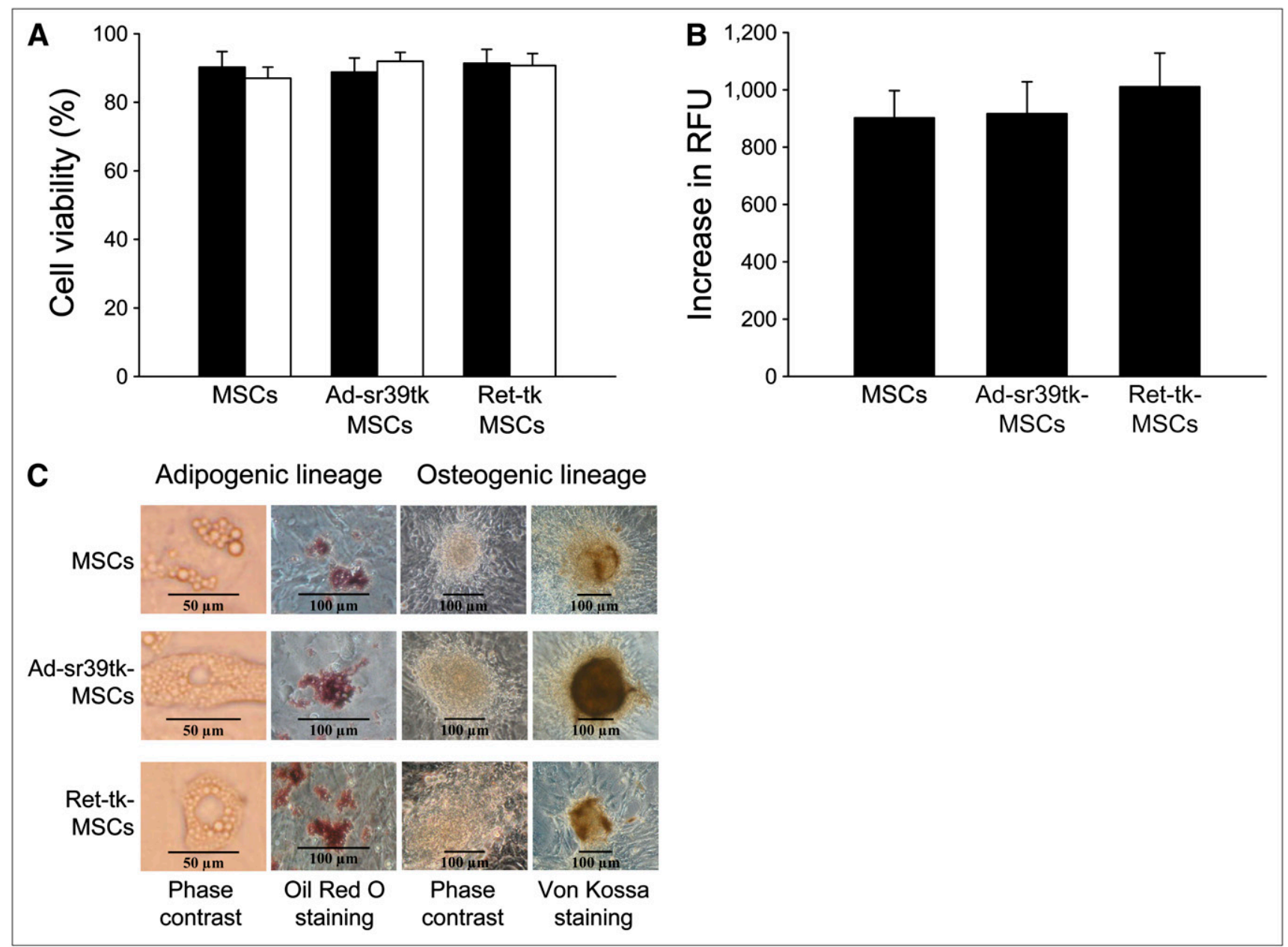

FIGURE 2. Effect of reporter gene on viability (trypan blue viability test, 24 [closed bars] and $48 \mathrm{~h}$ [open bars] after plating) (A), proliferation (DHL cell proliferation assay) (B), and differentiation (C) of MSCs. RFU = relative fluorescence units.

ing tk. The cellular uptake of ${ }^{3} \mathrm{H}-\mathrm{PCV}$ in Ad-sr39tk-MSCs and Ret-tk-MSCs increased in a dose-dependent manner. However, when results are expressed in percentage of total ${ }^{3} \mathrm{H}-\mathrm{PCV}$ activity, the relative ${ }^{3} \mathrm{H}-\mathrm{PCV}$ conversion was independent of the dose of ${ }^{3} \mathrm{H}-\mathrm{PCV}$ added to the culture medium (Fig. 4B).

In noninfected MSCs, no significant tk activity could be detected $\left({ }^{3} \mathrm{H}-\mathrm{PCV}\right.$ incorporation, $0.49 \% \pm 0.17 \% / \mathrm{mg}$ of protein). By contrast, tk activity was found to be significantly increased in both Ad-sr39tk-MSCs and Ret-tk-MSCs $(6.3 \% \pm 3.8 \% / \mathrm{mg}$ of protein vs. $5.0 \% \pm 2.5 \% / \mathrm{mg}$ of protein, respectively; $P<0.001$ vs. noninfected MSCs), with a nonsignificant trend toward a higher ${ }^{3} \mathrm{H}-\mathrm{PCV}$ accumulation in Ad-sr39tk-MSCs than in Ret-tk-MSCs.

\section{Time Course of tk Activity After Adenoviral and Retroviral Infection}

As shown in Figure 5A, tk activity peaked at $24 \mathrm{~h}$ after adenoviral infection and then slowly declined. Figure 5B demonstrates a higher tk activity in noninfected control MSCs after retroviral infection during the first 2 mo after infection. One month later, the tk activity of Ad-sr39tkMSCs and Ret-tk-MSCs was no longer different from that in noninfected control MSCs $(0.79 \% \pm 0.05 \% / \mathrm{mg}$ of protein vs. $0.67 \% \pm 0.06 \% / \mathrm{mg}$ of protein).

\section{In Vitro and In Vivo PET of MSCs}

First, we investigated the detection limits of our PET camera. For this purpose, we imaged infected cells that were cultured on 12-well dishes and incubated in advance with ${ }^{18} \mathrm{~F}$-FHBG. The minimal number of labeled cells that could clearly be visualized in vitro was 10,000 .

Next, we evaluated the ability of our PET system to track infected MSCs that were incubated in advance with ${ }^{18} \mathrm{~F}$ FHBG. To this end, prelabeled Ad-sr39tk-MSCs or Ret-tkMSCs were injected into the tibial anterior muscle of rats. Small-animal PET was performed at $30 \mathrm{~min}$ and $4 \mathrm{~h}$ after injection (Fig. 6A). In each instance, the PET images demonstrated the presence of a hot spot at the site of MSC injection, the intensity of which correlated strongly with the activity measured directly in the muscle by well counting $(r=0.99, P<0.0001)$. As shown in Figure 6B, the activity 


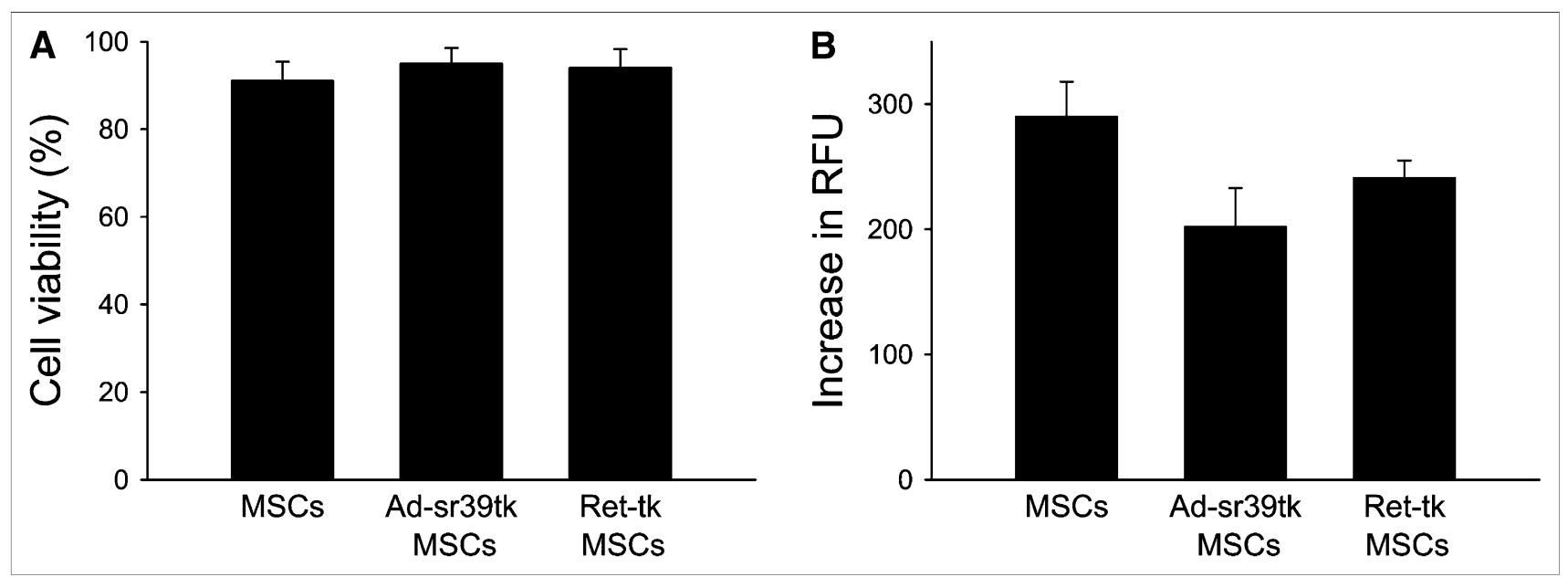

FIGURE 3. Effect of reporter probe viability (trypan blue viability test) (A) and proliferation (DHL cell proliferation assay) (B) on MSCs. RFU = relative fluorescence units.

measured by PET at the site of MSC injection correlated well with the number of injected prelabeled Ad-sr39tkMSCs.

Finally, we tested the possibility of imaging nonlabeled infected MSCs after the intravenous injection of the PET reporter probe ${ }^{18} \mathrm{~F}-\mathrm{FHBG}$. For this purpose, $1 \times 10^{6}$ Adsr39tk-MSCs or Ret-tk-MSCs were injected into the triceps brachialis muscle. At $24 \mathrm{~h}$ later, the animals received an intravenous administration of ${ }^{18} \mathrm{~F}-\mathrm{FHBG}(37 \pm 4 \mathrm{MBq})$. A PET scan was performed $3 \mathrm{~h}$ later. As with the prelabeled MSCs, the PET images demonstrated the presence of a hot spot at the site of MSC injection (Fig. 7).

\section{DISCUSSION}

To evaluate the success of stem cell transplantation and to monitor its outcome longitudinally in living individuals, the development of noninvasive imaging modalities capable of identifying the location, magnitude, and duration of cellular survival and fate is required. Recently, investigators have developed a reporter gene imaging method that allows transplanted stem cells to be imaged noninvasively using ${ }^{18}$ F-FHBG and PET $(6,11,13)$. The method uses HSV1-tk or its mutant derivative (HSV1-sr39tk) as a reporter gene. These genes are generally transduced in the cell lines of interest using adenoviral vectors; unfortunately, however, the expression of the transgene lasts only for a few days using adenoviruses, which is a severe limitation for tracking stem cell survival and proliferation longitudinally. Because of these drawbacks, in the present study we investigated the possibility of using retroviruses instead of adenoviruses to induce more stable transgene expression and observed that MSCs infected with either adenoviruses or retroviruses carrying the HSV1-tk gene can be useful for probing tk enzyme activity. In adenovirus-infected MSCs, tk enzyme activity peaks at $24 \mathrm{~h}$ after infection and then rapidly declines. By contrast, in retrovirus-infected MSCs,

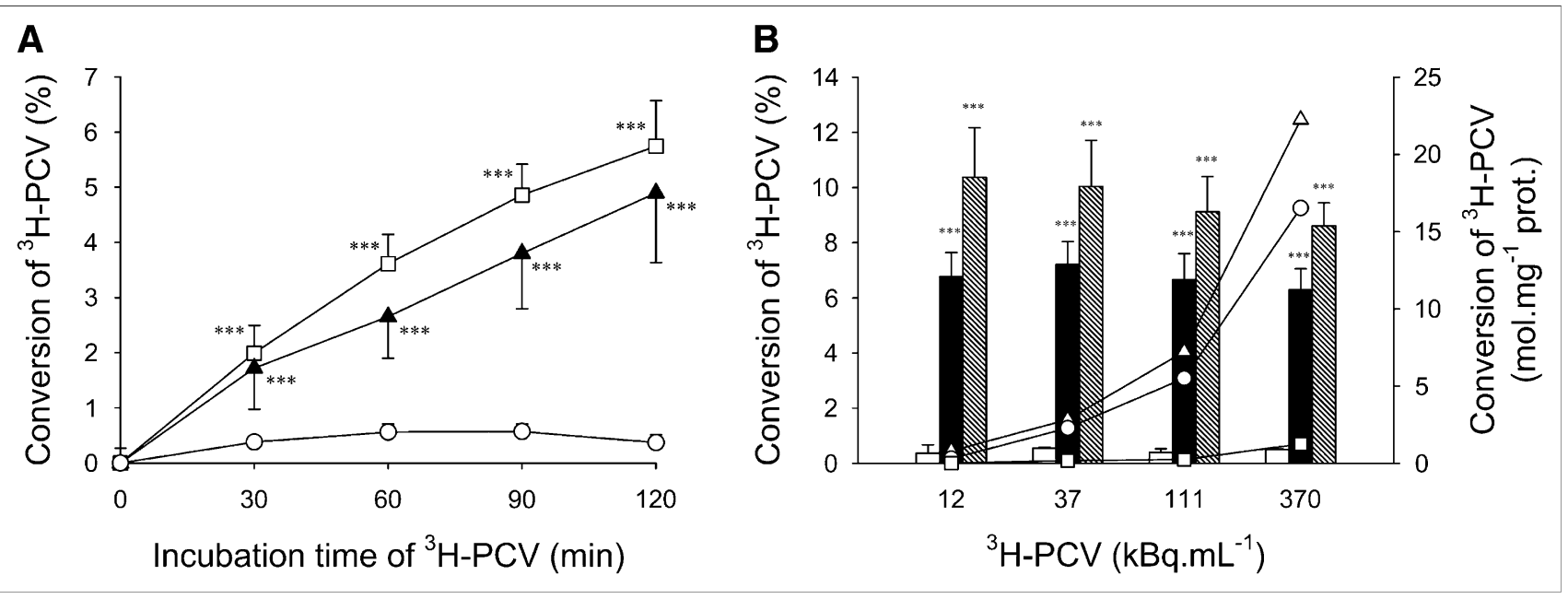

FIGURE 4. Time course $(\mathrm{A})$ and dose response $(\mathrm{B})$ of ${ }^{3} \mathrm{H}-\mathrm{PCV}$ cellular uptake in noninfected MSCs (O/open bars), Ret-tk-MSCs ( $\mathbf{\Lambda}$ /solid bars), and Ad-sr39tk-MSCs ( $\square$ /hatched bars). ${ }^{\star \star \star} P<0.001$ vs. noninfected MSCs. prot. $=$ protein. 


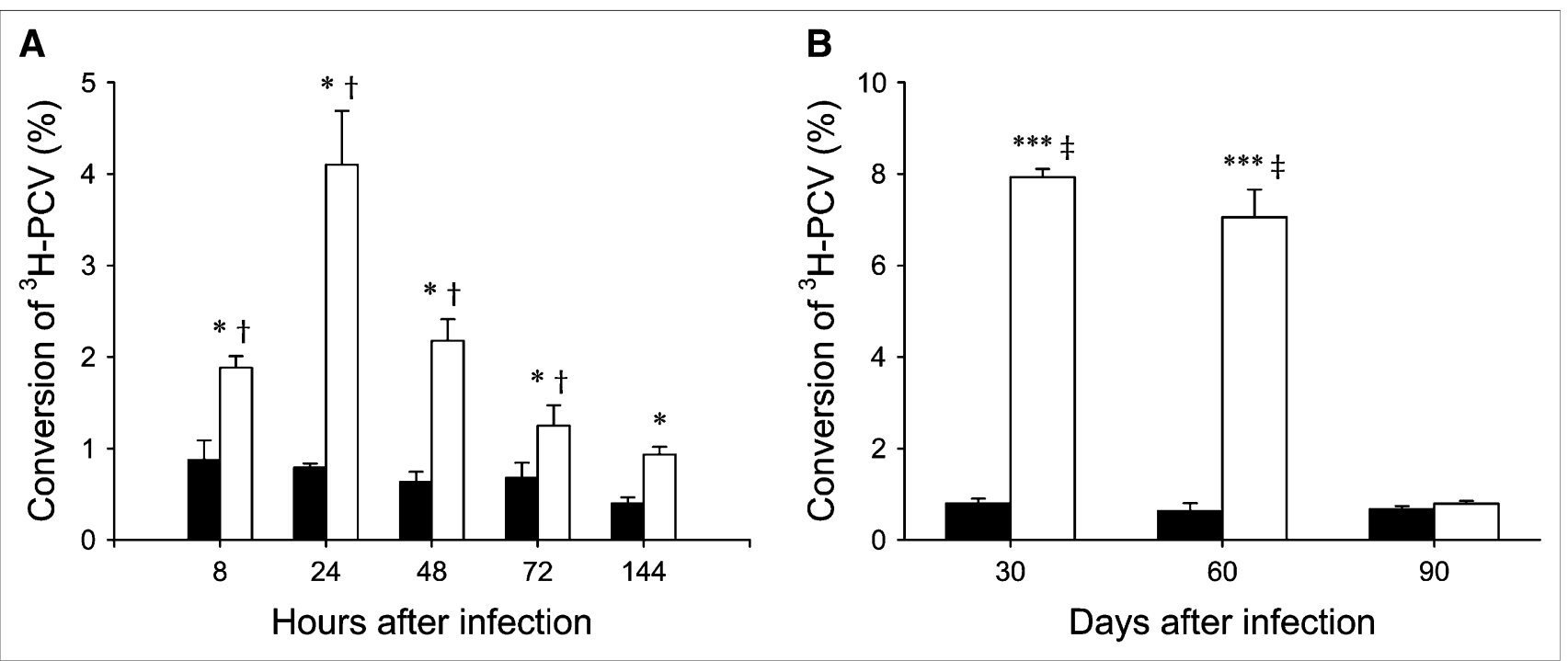

FIGURE 5. Time course of tk activity after infection. (A) Time course of percentage ${ }^{3} \mathrm{H}-\mathrm{PCV}$ conversion in noninfected MSCs (solid bars) and Ad-sr39tk-MSCs (open bars). ${ }^{*} P<0.05$ vs. MSCs; ${ }^{\dagger} P<0.05$ vs. $144 \mathrm{~h}$. (B) Time course of percentage ${ }^{3} \mathrm{H}-\mathrm{PCV}$ conversion in noninfected MSCs (solid bars) and Ret-tk-MSCs (open bars). ${ }^{\star \star \star} P<0.001$ vs. MSCs; ${ }^{*} P<0.001$ vs. 90 d.

tk enzyme activity remains stable for about $2 \mathrm{mo}$, after which, under our experimental conditions, it starts declining. In addition, neither adenovirus infection nor retrovirus infection significantly modifies MSC phenotype, viability, proliferation, and differentiation capabilities, and exposure of transduced MSCs to the reporter probe ${ }^{18} \mathrm{~F}-\mathrm{FHBG}$ does not alter the viability or proliferation rate of MSCs. Finally, a certain amount of the MSCs infected with either adenoviruses or retroviruses carrying the HSV1-tk gene or its variant can be imaged in vivo after their transplantation into the muscle of a living rat using ${ }^{18} \mathrm{~F}-\mathrm{FHBG}$ and small-animal PET.

\section{Effects of Adenoviral and Retroviral Infections on MSC Phenotype, Viability, Proliferation, and Differentiation Capabilities}

Because retroviruses integrate their genetic material at random into the host cell genome, they have the potential to modify the phenotype, viability, or proliferation capabilities
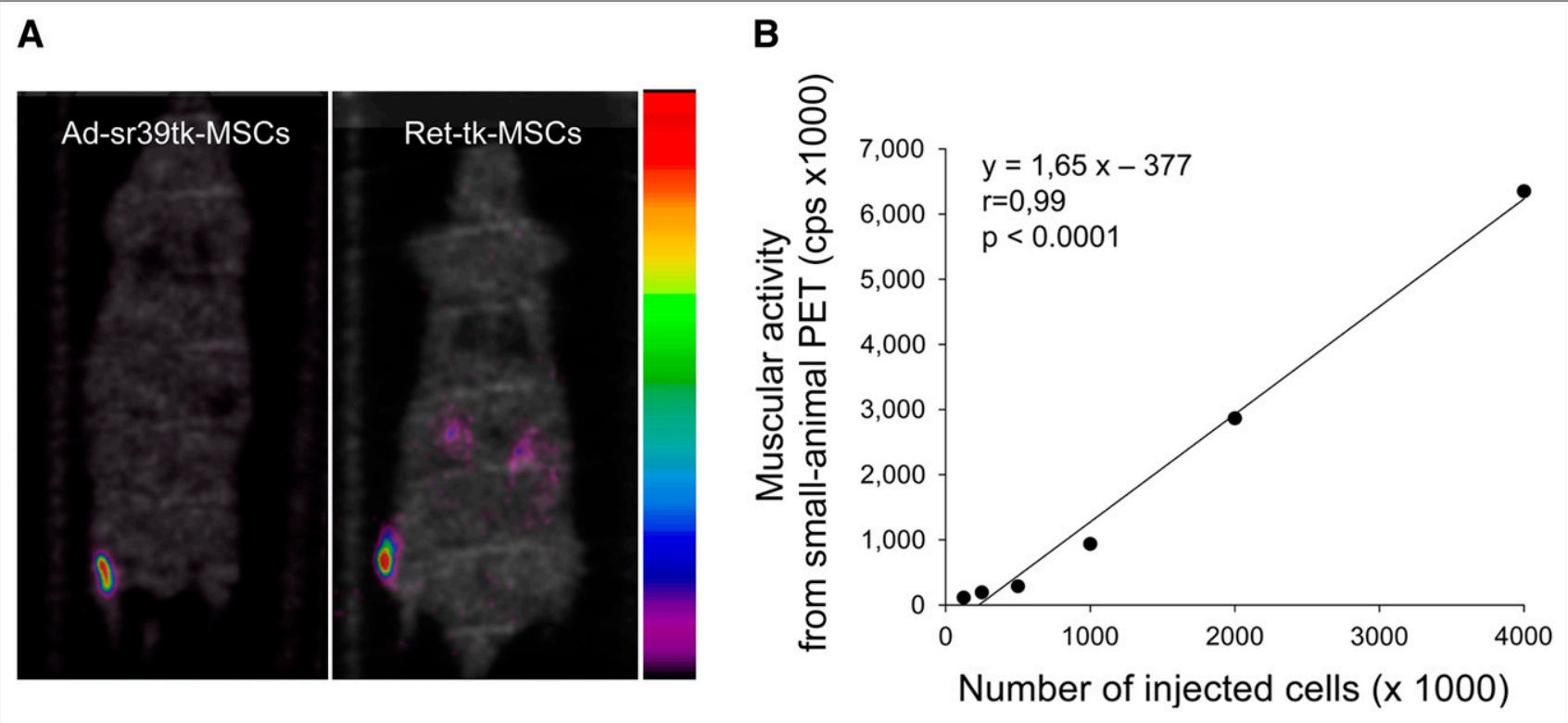

FIGURE 6. (A) Color-coded coronal emission images obtained at $4 \mathrm{~h}$ after intramuscular injections of ${ }^{18} \mathrm{~F}-\mathrm{FHBG}$-prelabeled Adsr39tk-MSCs and Ret-tk-MSCs. Emission images are superimposed on inverted gray-scaled coronal transmission images. (B) Correlation between muscular activity measured on small-animal PET images and number of transplanted Ad-sr39-tk-MSCs incubated in advance with ${ }^{18} \mathrm{~F}-\mathrm{FHBG}$. 


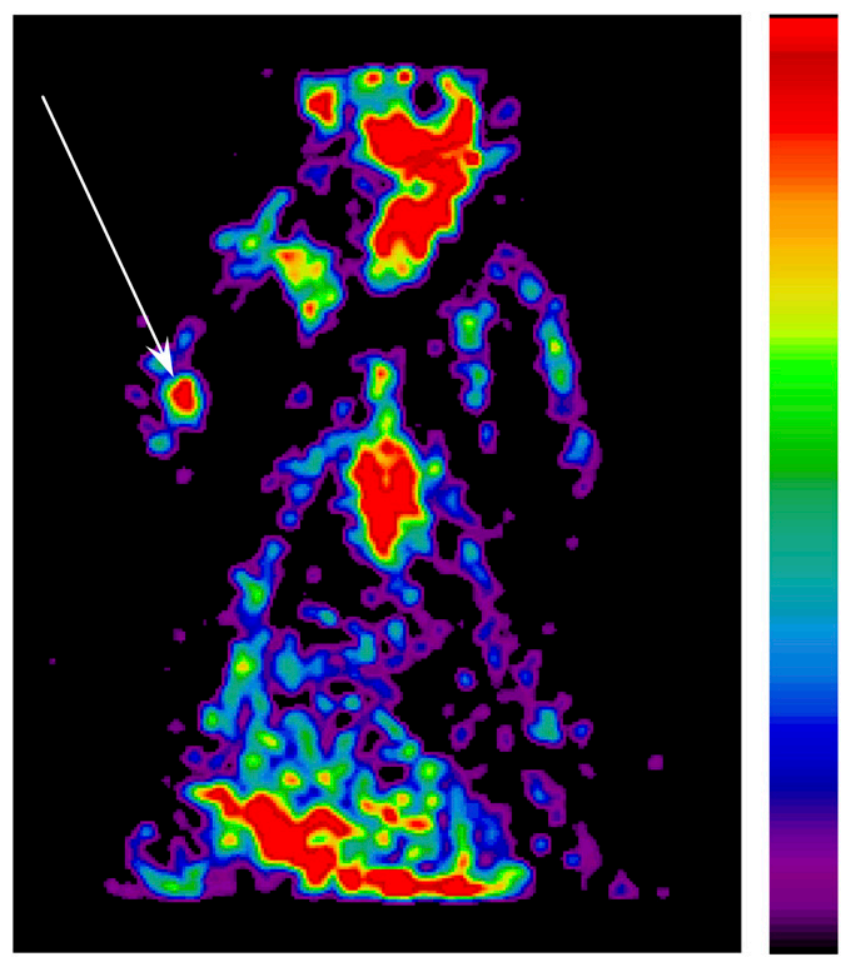

FIGURE 7. Color-coded coronal emission image obtained $3 \mathrm{~h}$ after intravenous injection of ${ }^{18} \mathrm{~F}-\mathrm{FHBG}(37 \pm 4 \mathrm{MBq})$. Hot spot (arrow) is clearly depicted in area of right elbow, into which $1 \times 10^{6} \mathrm{Ad}$-sr39tk-MSCs were injected day before.

of the infected cells. To test this, we evaluated the expression of MSC-specific surface antigens in infected and noninfected cell populations and were unable to demonstrate any significant differences among the different cells types. Similarly, cell viability, proliferation, and differentiation capabilities were unaffected by either type of viral infection. These data are in agreement with those previously reported in mouse embryonic stem cells and isolated lymphocytes $(14,15)$. Future studies should look at gene-expression pattern and proteomic changes after cell infection as previously reported (16).

\section{Effect of Reporter Probe on Viability and Proliferation of Ad-sr39tk-MSCs and Ret-tk-MSCs}

Because positrons and high-energy photons produced by the disintegration of ${ }^{18} \mathrm{~F}$ can induce cellular damage, we evaluated the short-term effects of radiation exposure on cellular viability and proliferation rate in control and infected MSCs. In accordance with previously published data in mouse embryonic stem cells (14), we did not observe any deleterious effects of the reporter probe ${ }^{18} \mathrm{~F}-\mathrm{FHBG}$ on the viability and proliferation rate of transduced MSCs.

\section{Adenovirus Versus Retrovirus HSV1-tk Gene Transduction in MSCs}

Although both adenoviruses and retroviruses can be used to transfer the HSV1-tk gene to MSCs, a direct comparison of the transduction efficiency of these 2 vectors has never been performed. To test this, we measured the ability of infected MSCs to incorporate ${ }^{3} \mathrm{H}-\mathrm{PCV}$, a labeled substrate of the HSV1-tk that accumulates in cells in proportion to the enzyme activity. As expected, both Ad-sr39tk-MSCs and Ret-tk-MSCs were able to accumulate ${ }^{3} \mathrm{H}-\mathrm{PCV}$. Interestingly, the ability of both cell types to incorporate ${ }^{3} \mathrm{H}-\mathrm{PCV}$ was found to be similar, which is a rather surprising finding because of the known greater affinity of the sr39 mutant tk for the 2 reporter probes, ${ }^{3} \mathrm{H}-\mathrm{PCV}$ and ${ }^{18} \mathrm{~F}-\mathrm{FHBG}(11,17)$. Although this finding remains speculative, the results were likely due to the relatively lesser susceptibility of MSCs to adenoviral infections. Whereas in most cell types adenoviral infections are associated with higher transduction efficiencies than other viral infections, this is usually not the case in MSCs. These cells express few adenovirus receptors on their surface, which in turn reduces their sensitivity to adenoviral infections (16). Alternatively, differences in transduction efficiency could also have been related to the use of different promoters in our adenoviral and retroviral constructs. Promoters represent critical elements that work in concert with other regulatory regions to direct the level of transcription of a given gene. Even if the CMV and the $5^{\prime}$ long terminal response promoters are both known to be strong promoters, we cannot exclude the possibility that, under our experimental conditions, they differentially affected transgene expression.

\section{Adenoviral and Retroviral Gene Silencing}

Because it leads only to episomal gene expression whereby the reporter gene is not integrated into the infected cells, gene silencing is a common phenomenon after adenoviral infection. Accordingly, the expression of the transgene is only transient, particularly in rapidly dividing cells, in which the expression of the transgene can last only for a few days. At variance with adenoviruses, retroviruses stably integrate their genetic material into the cell chromatin. Thus, in principle, they should allow for a more prolonged and more stable transgene expression and hence for the longitudinal tracking of the transduced cells. Yet recognition and inactivation of the alien genes by the target cells is frequently encountered after retroviral infection. The mechanisms underlying this phenomenon are varied and still poorly understood $(18,19)$. Retroviral gene silencing probably explains why the cellular uptake of ${ }^{3} \mathrm{H}-\mathrm{PCV}$ began to decrease 2 mo after infection in our experiments. Alternatively, the decrease in cellular uptake of ${ }^{3} \mathrm{H}-\mathrm{PCV}$ could have been due to progressive dilution of the retrovirally infected clones by noninfected clones. Indeed, we did not supplement the culture medium with G-418 sulfate at any time during the whole 3-mo culture period. Accordingly, we cannot exclude the possibility that dilution of the infected cell population by noninfected cells led to an apparent decrease in HSV1-tk expression over time.

\section{Imaging of MSCs After Adenoviral or Retroviral Reporter Gene Transduction}

Because the tissular retention of transplanted MSCs depends on several factors that are still far from being 
understood, we concentrated our efforts mostly on investigating the feasibility of imaging prelabeled MSCs in vivo using small-animal PET and on defining the detection limits of the small-animal PET instrument. Our data show that Ad-sr39tk-MSCs and Ret-tk-MSCs, incubated for $60 \mathrm{~min}$ with ${ }^{18} \mathrm{~F}-\mathrm{FHBG}$, can be readily imaged with a small-animal PET camera, that the activity measured by the PET camera closely parallels the number of injected cells, and that the instrument allows for a minimum of 10,000 labeled cells to be detected, which is consistent with previous reports $(20,21)$. Finally, our data show that infected MSCs can be traced after an intravenous injection of the PET reporter probe, irrespective of the vector used for transduction. Consequently, it should be possible to follow the fate of these cells over time using this approach.

Our study has limitations that should be acknowledged. First, to assess HSV1-tk gene expression, we assayed tk enzyme activity by measuring the rate of incorporation of ${ }^{3} \mathrm{H}-\mathrm{PCV}$ by the infected cell population. To do this, the cells were incubated with the labeled substrate for $60 \mathrm{~min}$. At the end of the incubation period, the medium was removed and total radioactivity was measured. Because total radioactivity represents the sum of both the phosphorylated and nonphosphorylated forms of the reporter probe ${ }^{3} \mathrm{H}-\mathrm{PCV}$, it only partially reflects HSV1-tk enzyme activity. However, because in noninfected MSCs incubated in the presence of ${ }^{3} \mathrm{H}-\mathrm{PCV}$ total radioactivity remained negligible, it is unlikely that significant amounts of nonphosphorylated ${ }^{3} \mathrm{H}-\mathrm{PCV}$ contributed to our results. Second, on the basis of our experiments, we cannot exclude the possibility that some of the MSCs that were exposed to the Ad-sr39tk vector were not effectively transduced. We feel, however, that this possibility is unlikely. Indeed, in preliminary experiments, we had exposed MSCs to an adenoviral construct carrying the LacZ gene driven by the CMV promoter. In those experiments, which were conducted in the same way as those reported in our current study, we observed that greater than $99 \%$ of the MSCs exposed to an MOI of 50 subsequently expressed the $\beta$-galactosidase enzyme.

\section{CONCLUSION}

Bone marrow-derived MSCs in rats can be infected with adenoviruses and retroviruses, permitting the expression of the tk PRG without altering the phenotype, viability, proliferation rate, and differentiation capabilities of these cells. Furthermore, infected MSCs can be noninvasively imaged using ${ }^{18} \mathrm{~F}-\mathrm{FHBG}$ and small-animal PET after transplantation into the tibial anterior muscle of living rats. The retroviral infection of MSCs should allow the fate of transplanted cells, until 2 mo after infection, to be followed.

\section{ACKNOWLEDGMENTS}

We are grateful to Isabelle de Hemptinne for sharing her experience of stem cell handling and Raymond Bausart for technical assistance. We were supported by the Fonds National de la Recherche Scientifique et Médicale (Belgium) and the Actions de Recherche Concertées (Belgium). This study was also supported by the Fund for Scientific Research in Industry and Agriculture, Belgium.

\section{REFERENCES}

1. Bartunek J, Croissant JD, Wijns W, et al. Pretreatment of adult bone marrow mesenchymal stem cells with cardiomyogenic growth factors and repair of the chronically infarcted myocardium. Am J Physiol Heart Circ Physiol. 2007; 292:H1095-H1104.

2. Li X, Yu X, Lin Q, et al. Bone marrow mesenchymal stem cells differentiate into functional cardiac phenotypes by cardiac microenvironment. J Mol Cell Cardiol. 2007;42:295-303.

3. Zimmet JM, Hare JM. Emerging role for bone marrow derived mesenchymal stem cells in myocardial regenerative therapy. Basic Res Cardiol. 2005;100:471-481.

4. Mangi AA, Noiseux N, Kong D, et al. Mesenchymal stem cells modified with Akt prevent remodeling and restore performance of infarcted hearts. Nat Med. 2003;9:1195-1201.

5. Pittenger MF, Martin BJ. Mesenchymal stem cells and their potential as cardiac therapeutics. Circ Res. 2004;95:9-20.

6. Wu JC, Chen IY, Sundaresan G, et al. Molecular imaging of cardiac cell transplantation in living animals using optical bioluminescence and positron emission tomography. Circulation. 2003;108:1302-1305.

7. Gardlik R, Palffy R, Hodosy J, Lukacs J, Turna J, Celec P. Vectors and delivery systems in gene therapy. Med Sci Monit. 2005;11:110-121.

8. Walther W, Stein U. Viral vectors for gene transfer: a review of their use in the treatment of human diseases. Drugs. 2000;60:249-271.

9. Hung SC, Deng WP, Yang WK, et al. Mesenchymal stem cell targeting of microscopic tumors and tumor stroma development monitored by noninvasive in vivo positron emission tomography imaging. Clin Cancer Res. 2005;11:7749-7756.

10. Love Z, Wang F, Dennis J, et al. Imaging of mesenchymal stem cell transplant by bioluminescence and PET. J Nucl Med. 2007;48:2011-2020.

11. Gambhir SS, Bauer E, Black ME, et al. A mutant herpes simplex virus type 1 thymidine kinase reporter gene shows improved sensitivity for imaging reporter gene expression with positron emission tomography. Proc Natl Acad Sci USA. 2000;97:2785-2790.

12. Di Ianni M, Di Florio S, Venditti G, et al. T-lymphocyte function after retroviralmediated thymidine kinase gene transfer and G418 selection. Cancer Gene Ther. 2000;7:920-926.

13. Gambhir SS, Barrio JR, Wu L, et al. Imaging of adenoviral-directed herpes simplex virus type 1 thymidine kinase reporter gene expression in mice with radiolabeled ganciclovir. J Nucl Med. 1998;39:2003-2011.

14. Cao F, Lin S, Xie X, et al. In vivo visualization of embryonic stem cell survival, proliferation, and migration after cardiac delivery. Circulation. 2006;113:1005-1014.

15. Munshi NC, Govindarajan R, Drake R, et al. Thymidine kinase (TK) genetransduced human lymphocytes can be highly purified, remain fully functional, and are killed efficiently with ganciclovir. Blood. 1997;89:1334-1340.

16. Conget PA, Minguell JJ. Adenoviral-mediated gene transfer into ex vivo expanded human bone marrow mesenchymal progenitor cells. Exp Hematol. 2000;28:382-390.

17. Min JJ, Iyer M, Gambhir SS. Comparison of $\left[{ }^{18} \mathrm{~F}\right] \mathrm{FHBG}$ and $\left[{ }^{14} \mathrm{C}\right] \mathrm{FIAU}$ for imaging of HSV1-tk reporter gene expression: adenoviral infection vs stable transfection. Eur J Nucl Med Mol Imaging. 2003;30:1547-1560.

18. Swindle CS, Klug CA. Mechanisms that regulate silencing of gene expression from retroviral vectors. J Hematother Stem Cell Res. 2002;11:449-456.

19. Bestor TH. Gene silencing as a threat to the success of gene therapy. J Clin Invest. 2000;105:409-411.

20. Fischer BM, Olsen MW, Ley CD, et al. How few cancer cells can be detected by positron emission tomography? A frequent question addressed by an in vitro study. Eur J Nucl Med Mol Imaging. 2006;33:697-702.

21. Su H, Forbes A, Gambhir SS, Braun J. Quantitation of cell number by a positron emission tomography reporter gene strategy. Mol Imaging Biol. 2004;6:139-148. 